\title{
Discours
}

Revue de linguistique, psycholinguistique et

informatique. A journal of linguistics, psycholinguistics

and computational linguistics

$2 \mid 2008$

Varia

\section{Sémantisme et potentiel argumentatif des dérivés dénominaux en anti-}

Franziska Heyna

URL : http://journals.openedition.org/discours/2022

DOI : 10.4000/discours.2022

ISSN : 1963-1723

Éditeur :

Laboratoire LATTICE, Presses universitaires de Caen

\section{Référence électronique}

Franziska Heyna, «Sémantisme et potentiel argumentatif des dérivés dénominaux en anti- 》, Discours [En ligne], 2 | 2008, mis en ligne le 11 octobre 2017, consulté le 02 mai 2019. URL : http:// journals.openedition.org/discours/2022 ; DOI : 10.4000/discours.2022

Ce document a été généré automatiquement le 2 mai 2019.

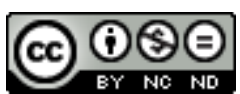

Discours est mis à disposition selon les termes de la licence Creative Commons Attribution - Pas d'Utilisation Commerciale - Pas de Modification 4.0 International. 


\title{
Sémantisme et potentiel argumentatif des dérivés dénominaux en anti-
}

\author{
Franziska Heyna
}

L'étude présentée ici se situe dans le prolongement d'un projet de recherche soutenu par le Fonds national suisse de la recherche scientifique (projet FNRS 12-61673.00: « Dérivations morphologiques et typage des entités sémantiques »). Je remercie très chaleureusement mes deux relecteurs : leurs remarques très pertinentes m'ont permis d'affiner plusieurs aspects de mon analyse.

Cette étude sera axée sur l'analyse du sémantisme des dérivés dénominaux en anti-; les antiA de type anticancéreux et antigrippal ne seront pas abordés. Par ailleurs, nous nous intéresserons uniquement aux bases substantivales simples, les antiN formés sur un substantif déverbal (anti-expulsion, anti-chasse) ne seront en principe pas pris en compte.

Les rares articles consacrés à la préfixation en anti- (Rey, 1968, Corbin 1987, Fradin 1997a $1997 b^{1}$ ) s'accordent à dégager deux valeurs sémantiques distinctes, illustrées par les deux occurrences suivantes :

[1] Il y a, dans Manhattan, plus de deux mille instituts de dermatologie, de salons anti-rides, de praticiens du cuir chevelu, masseurs, ondulateurs et chirurgiens plastiques, spécialistes de l'excision du double menton, [...]. [f, MORAND]

[2] Scandaleux Mercutio, conteur de balivernes, antihéros que n'anime nul esprit de sacrifice non plus qu'aucune dévotion à quelque cause juste ou injuste, mais qui se risque par jeu ! [f, LEIRIs].

En [1], anti- prend une valeur adversative et le antiN se glose par « qui est contre la notion désignée par $\mathrm{N} »$; en [2], anti- traduit une valeur antonymique et le dérivé se paraphrase par «qui est le contraire de $\mathrm{N}$ ». Par commodité, les chiffres indexés à la suite du morphème anti- seront utilisés pour référer aux valeurs respectivement adversative et antonymique, telles qu'elles apparaissent dans antirides (anti ${ }_{1}^{-}$) et dans antiroman (anti $i_{2}^{-}$). Mis à part Rey (1968), qui consacre un paragraphe à la description sémique des anti ${ }_{2} \mathrm{~N}$, il 
n'existe à notre connaissance pas d'étude dédiée aux formations $\operatorname{anti}_{2} \mathrm{~N}$ : après signalement de deux valeurs sémantiques distinctes, les auteurs s'intéressent aux seuls emplois adversatifs de anti-.

Notre approche s'appuie sur des faits empiriques en contexte, afin de mettre en lumière certaines difficultés interprétatives ${ }^{2}$. Très sommairement, il est possible de soutenir que les difficultés interprétatives pour les anti ${ }_{1} \mathrm{~N}$ ont trait au statut 'référentiel' ambigu de $\mathrm{N}$, alors que dans le cas des $a_{n} i_{2} \mathrm{~N}$, c'est plutôt au niveau de la portée du préfixe qu'elles se situent. Au-delà d'une description fine des deux valeurs sémantiques, l'intérêt de la présente étude est d'examiner le type d'objets-de-discours sélectionné par anti- (individus discrets, noms génériques, etc.), et de déterminer les possibilités argumentatives qui en découlent. Avant d'aborder les analyses sémantiques et discursives, quelques préalables d'ordre morphosyntaxique seront proposés.

\section{Propriétés morphosyntaxiques des dérivés anti ${ }_{1} \mathrm{~N}$ et $\operatorname{anti}_{2} \mathrm{~N}$}

\subsection{La distribution des dérivés anti ${ }_{1} \mathrm{~N}$ et anti ${ }_{2} \mathrm{~N}$}

Sur le plan distributionnel, les formations antiN se distribuent dans les trois contextes syntaxiques suivants :

(i) en position d'épithète (contexte $\alpha$ ) :

[3] Selon les associations anti-expulsion, environ 25 enfants sont menacés d'expulsion à la fin de l'année scolaire dans la région nantaise, [...]. [w]

(ii) en position de tête de syntagme (contexte $\beta)$ :

[4] Les antirides envahissent les rayons de cosmétiques masculins,... [p, Libération, 23 août 2005]

[5] Avec Bouvard et Pécuchet, Flaubert a choisi d'écrire un antiroman, où des antihéros, parangons de la bêtise, se livrent à une quête de la vérité chaque fois recommencée et qui chaque fois s'achève en catastrophe. [w]

(iii) en position d'attribut (contexte $\gamma)$ :

[6] Pour Bill Gates, le format Blu-Ray est anti-consommateur. [w]

Alors que les dérivés en anti ${ }_{1}$ (valeur adversative) figurent dans les trois contextes $\alpha$, $\beta$ et $\gamma$, les anti ${ }_{2} \mathrm{~N}$ (valeur antonymique) entrent principalement, mais non exclusivement, dans le contexte « tête de $\mathrm{SN}$ ». Les dérivés formés avec le préfixe anti ${ }_{1}$ - ont préférentiellement comme rendement la qualification, tandis que les dérivés formés avec anti ${ }_{2}$ - fonctionnent au contraire comme des prédicats catégorisateurs qui permettent de dénoter un objet-dediscours (Berrendonner, 1995). La présence d'un modifieur de type très ou assez ${ }^{3}$ corrobore l'hypothèse que les anti ${ }_{1} \mathrm{~N}$ fonctionnent comme qualificatifs et dénotent des propriétés conçues comme graduables:

[7] Toutes les religions, sans exception, sont fondamentalement anti-femmes . [p, L'Express, 6 juin 2005]

[8] Il n'y a pas de doctrine tellement établie sur les guillemets. D'ailleurs, je suis moi-même assez anti-guillemets. [b2, Actuel, novembre 1989] 
On peut être plus ou moins anti-femmes, comme on peut être plus ou moins anti-guillemets ...

Si un dérivé de type anti ${ }_{2} \mathrm{~N}$ occupe la position d'attribut, deux cas de figure se présentent :

(i) Soit le dérivé maintient sa valeur antonymique :

[9] <à propos d'une BD manga> Voilà une série hors du commun, le héros Dark Schneider est totalement antihéros, cruel, sadique, pervers et sans aucune moralité. [w]

Cette interprétation est corroborée par la suite d'adjectifs (cruel, sadique, etc.) qui dénotent autant de facettes justifiant le qualificatif d'antihéros. Le statut de qualificatif du dérivé antihéros est d'ailleurs confirmé par la présence d'un modifieur (totalement).

(ii) Soit le dérivé anti ${ }_{2} \mathrm{~N}$ est réinterprété comme un dérivé anti ${ }_{1} \mathrm{~N}$ à valeur adversative :

[10] Je crois que mon ordi est antiroman car je viens juste de réécrire celui que je vous ai écrit hier et rebelote monsieur refait des siennes. [w]

Le dérivé antiroman a lexicalisé le sens antonymique. En [10] par contre, le dérivé s'interprète par «qui est hostile au(x) roman(s) ", interprétation qui est, là encore, confirmée par le contexte.

L'exemple suivant est quant à lui parfaitement ambigu : a-t-on affaire à un anti ${ }_{2} \mathrm{~N}$ à valeur antonymique ou à un anti ${ }_{2} \mathrm{~N}$ réinterprété comme un anti ${ }_{2} \mathrm{~N}$ à valeur adversative?

[11] < Sandra Kim> En fait, je suis très anti-star. De ce point de vue, je suis un peu la « Goldmann belge », qui a toujours pris du recul par rapport à tout le star system [...]. [w]

Faut-il interpréter " prendre du recul par rapport au star system » comme une propriété caractéristique de quelqu'un qui est « une anti-star », ce qui parlerait en faveur d'une interprétation à valeur antonymique, ou comme une glose de quelqu'un qui est « opposé aux stars ", ce qui correspondrait à la valeur adversative ? Pour cet exemple, le contexte ne permet pas de trancher entre les deux lectures.

La distribution des dénominaux en anti- est résumée ci-après :

\begin{tabular}{|c|c|c|}
\hline & anti $_{1} \mathrm{~N}$ (valeur adversative) & anti $2 \mathrm{~N}$ (valeur antonymique) \\
\hline $\begin{array}{l}\text { a. [Dét. } \mathrm{N}-\text { - Jsa.... } \\
(=\text { fonction "epithete })\end{array}$ & $\begin{array}{l}\text { les associations anti-expulsion } \\
\text { (3) }\end{array}$ & $? !$ \\
\hline $\begin{array}{l}\text { B. [Dét - - dsi:... } \\
(=\text { fonction } \& \text { tête de SN } »)\end{array}$ & Les antirides... (4) & $\begin{array}{l}\ldots \text { un antiroman,... des } \\
\text { antihéros }(5)\end{array}$ \\
\hline $\begin{array}{l}\gamma . \text { être - } \\
(=\text { fonction } * \text { attribut } s)\end{array}$ & $\begin{array}{l}\text {... est anti-consommateur (6); } \\
\text { mon ordi est antiroman (10) }\end{array}$ & ... est antihéros (9) \\
\hline
\end{tabular}

Tableau 1. La distribution des dérivés ANTI, $N$ et $\mathbf{A N T I}_{\mathbf{2}} \mathrm{N}$

\subsection{Les anti, $\mathrm{N}$ et anti ${ }_{2} \mathrm{~N}$ - des noms (substantifs-adjectifs)}

A la suite des travaux de Noailly (1990, 1999), nous considérons que substantifs et adjectifs appartiennent à une seule catégorie grammaticale qui est celle des noms ${ }^{4}$. Les éléments de cette catégorie, les lexèmes nominaux, possèdent des emplois syntaxiques préférentiels et statistiquement majoritaires, ce qui revient à dire que l'opposition 
substantif vs adjectif est une opposition graduelle. Pour les antiN, on peut observer les tendances distributionnelles suivantes, au vu des exemples de notre corpus :

- les anti ${ }_{2} \mathrm{~N}$ sont presque exclusivement employés en position de tête de SN et se trouvent par conséquent à l'extrémité gauche du continuum substantifs-adjectifs : ce sont des $\mathrm{N}$ employés préférentiellement comme substantifs ;

- à l'extrémité droite figurent les anti ${ }_{1} \mathrm{~N}$, employés préférentiellement comme épithète ou attribut, mais qui peuvent également être utilisés syntaxiquement comme substantifs; certains anti ${ }_{1} \mathrm{~N}$ comme antigrippe ou antiride se sont lexicalisés comme substantifs et sont passés du statut de qualificatif à celui de catégorisateur.

\begin{tabular}{|c|c|}
\hline 4 ४⿻一丿八 & $\mathrm{N}$ adjectifs \\
\hline $\begin{array}{l}\text { (un) antiroman; (un) anti-collier } \\
\text { (uns) antiride(s); (un) antimitels }\end{array}$ & $\begin{array}{r}\text { anti-expulsion } \\
\text { anti-consommateur }\end{array}$ \\
\hline $\begin{array}{l}\text { antij, } \\
\text { antipl } \text { lexicalisés }\end{array}$ & $a n t_{3} N$ \\
\hline
\end{tabular}

Tableau 2. Le continuum des substantifs-adjectifs en antiN

\subsection{Les niveaux d'analyse pris en compte}

Dans ce qui suit, nous distinguerons trois niveaux d'analyse :

- Un niveau segmental, avec les notations $a n t i_{1}-$ / anti $i_{2}$ - et $\mathrm{N}$ pour la base substantivale.

- Un niveau sémantique : le sens lexical d'un mot construit sera décrit comme l'application d'un ou de plusieurs opérateurs à un opérande pour produire un résultat R'. Les préfixes anti $i_{1}^{-}$et anti $i_{2}$ - sont les variables sur le plan segmental qui font office d'opérateurs; ils seront abrégés $O P_{-} A N T I_{1}$ et $O P_{-} A N T I_{2}$. Les substantifs fonctionnent comme opérandes; en tant qu'opérandes, ils seront suivis de l'indice « prime » $\left(\mathrm{N}^{\prime}\right)$.

- Au niveau discursif, nous parlerons d'objets-de-discours, notés entre chevrons $\left(<\mathrm{N}>,<a n t i_{1}\right.$ $\left.\mathrm{N}>,<\operatorname{anti}_{2} \mathrm{~N}>\right)$.

Ces trois niveaux d'analyse sont schématiquement illustrés ci-après :

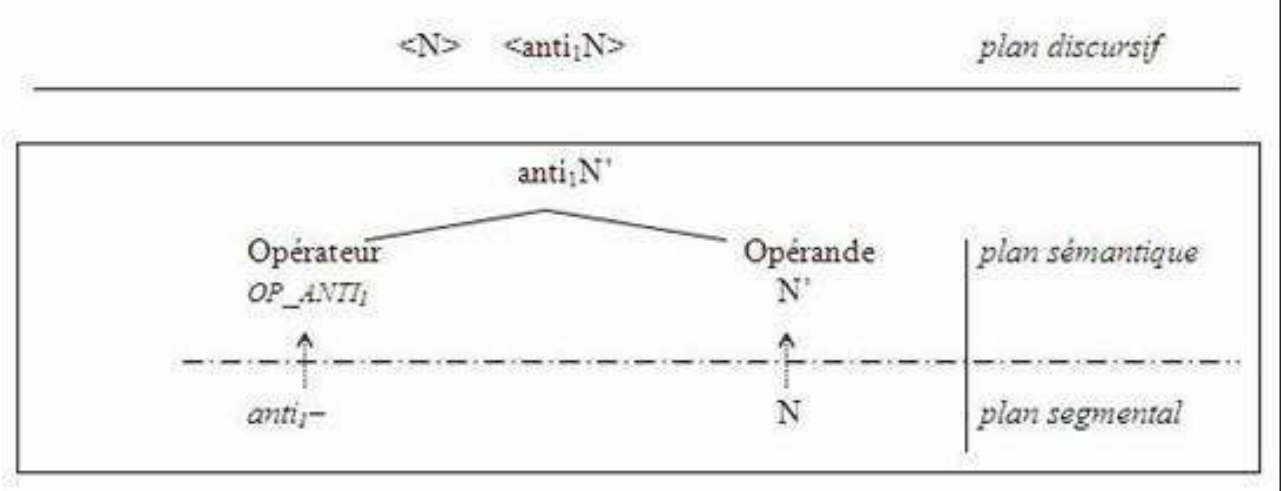

Tableau 3. Niveaux d'analyse 


\section{Sémantisme et potentiel argumentatif des dérivés anti ${ }_{1} \mathrm{~N}$}

\subsection{Valeurs sémantiques des dérivés anti ${ }_{1} \mathrm{~N}$ et anti ${ }_{2} \mathrm{~N}$}

\subsubsection{Valeur adversative (anti $\left.{ }_{1}^{-}\right)$et de valeur antonymique (anti $\left.{ }_{2}^{-}\right)$}

Dans la littérature, on s'accorde à distinguer deux valeurs sémantiques distinctes pour le préfixe anti-, valeurs qui se traduisent par les étiquettes suivantes : opérateur d'opposition vs opérateur antonymique chez Rey (1968: 54-55), antagonisme vs antonymie ou opposition polémique vs opposition descriptive ${ }^{5}$ chez Corbin (1980: 196), emplois oppositifs vs antipodaux ${ }^{6}$ chez Fradin (1997a : 90). Selon Noailly (1999 : 40), les anti ${ }_{1} \mathrm{~N}$ exprimeraient une opposition marquée par le trait [+actif], et les anti ${ }_{2} \mathrm{~N}$ une opposition marquée [-actif], sans que l'auteur précise plus en détail comment il faut comprendre les traits [ \pm actif]. Pour notre part, nous adopterons les termes de valeur adversative (anti $\left.1^{-}\right)$et de valeur antonymique (anti ${ }_{2}$ ).

Les différentes étiquettes recensées tentent toutes tant bien que mal d'exprimer une même différence entre les deux valeurs, différence qui se ramène à l'expression d'une « opposition active " vs une " opposition non active » : une crème antirides agit contre les rides, alors qu'un antiroman ne fait que manifester un écart avec le roman prototypique dont il tente de se démarquer. Cette différence de fonctionnement, pourtant immédiatement perceptible, n'est pas facilement modélisable.

Jusqu'à présent les chiffres indexés à la suite du morphème anti- ont été utilisés pour signaler deux valeurs sémantiques distinctes. On ne peut à présent éviter de prendre position quant au rapport entre ces « deux » morphèmes. Le rapport sémantique entre anti $i_{1}$ - et anti $i_{2}$ - est à notre avis trop évident et trop étroit pour avancer l'hypothèse de deux préfixes distincts, tous les deux exprimant une relation d'opposition. En revanche, il s'agira de trouver une explication pour rendre compte des deux types de réalisations, valeurs adversative $v s$ antonymique.

En guise de première approche, nous ferons l'hypothèse que les préfixes anti $i_{1}$ - et anti $i_{2}$ - ne se distinguent pas tant par une valeur sémantique différenciée, que par le type d'opérande sur lequel ils opèrent: dans les anti ${ }_{1} \mathrm{~N}$, anti- opérerait sur des entités concrètes, réelles, c'est-à-dire des extensions (mites, rides, ours, ...), d'où la paraphrase « qui s'oppose à »; dans les anti ${ }_{2} \mathrm{~N}$ en revanche, anti- opèrerait sur des entités abstraites, caractérisées par un certain nombre de traits sémantiques et donc des intensions (héros, pièce, roman, ...). Les traits sémantiques [士actif] ou encore les épithètes polémique vs descriptif attribuées à anti $i_{1}$ - et anti ${ }_{2}$ - trouveraient ainsi une explication bienvenue, chaque préfixe opérant un type d'extraction sémantique particulier: l'une portant sur des extensions (les anti $\left.{ }_{1} \mathrm{~N}\right)$, l'autre sur des intensions (les anti ${ }_{2} \mathrm{~N}$ ).

Le préfixe anti ${ }_{2}$ - agit effectivement uniquement sur l'intension de N (cf. infra, § 3.3); par contre, il n'est pas possible d'affirmer que anti $i_{1}^{-}$opère toujours sur des extensions. Considérons à ce propos les exemples suivants :

[12] <à propos de Simone Veil> Pour Mitterand, elle représente l'ouverture au centre. Pour Chirac, une caution morale anti-Le Pen. [b2, Le Point, 16 avril 1988] 
[13] Aux propriétés anti-ride et raffermissantes elle est idéale pour les peaux manquant de fermeté. [w]

En [12], anti- prend comme opérande un nom propre qui désigne un référent particulier. Le nom propre dans le dérivé anti $\mathrm{i}_{1} \mathrm{~N}$ remplit sa fonction référentielle (Jonasson, 19947) et fonctionne donc comme désignateur rigide au sens de Kleiber (1981). Pour [13], on ne peut soutenir que ride dénote un objet unique, extensionnel; dans cet exemple, <ride>, substantif singulier sur le plan segmental, désigne bien un objet-de-discours catégorisé comme un "nom générique " et donc un objet intensionnel. Comme nous allons le montrer, l'opposition binaire - objets extensionnels vs objets intensionnels - se révèle insuffisante pour décrire le fonctionnement des anti ${ }_{1} \mathrm{~N}$ et des anti ${ }_{2} \mathrm{~N}$. Une brève présentation de l'ontologie formelle du français s'avère donc nécessaire.

\subsubsection{L'ontologie formelle du français selon Berrendonner $(2002,2004)$}

Il existe dans l'ontologie formelle du français une catégorie d'objets - les types (Berrendonner, 2002, 2004) - qui appartiennent au domaine intensionnel, tout en servant à nommer des objets-de-discours ${ }^{8}$. Contrairement à ce qui est présumé dans le modèle frégéen (opposition binaire: termes vs prédicats), l'ontologie formelle du français serait triadique et comporterait trois catégories d'entités logico-cognitives :

\begin{tabular}{c|c|c|}
\hline \multicolumn{2}{|c|}{ Objets } & Fonctions \\
\hline $\begin{array}{c}\text { Realia } \\
\begin{array}{c}\text { Unlece panda a encore mordu } \\
\text { le gardien. }\end{array}\end{array}$ & $\begin{array}{c}\text { Types } \\
\text { Le panda est un herbivore. } \\
\text { cercle carré, licome }\end{array}$ \\
\hline $\begin{array}{c}\text { Extensionnel } \\
\text { Existence in re }\end{array}$ & \multicolumn{2}{|c|}{$\begin{array}{c}\text { Intensionnel } \\
\text { Existence in intellectu }\end{array}$} \\
\hline
\end{tabular}

Tableau 4. Ontologie formelle du français (d'après Berrendonner, 2002)

Les realia, présentées comme des entités existant in re, appartiennent au domaine extensionnel, alors que les types et les concepts, conçus comme des objets idéels, relèvent du domaine intensionnel. Les types se distinguent des concepts par le fait qu'ils dénotent des objets, alors que les concepts sont assimilables à des fonctions. Présentant des propriétés partagées avec les realia, les types sont figurés comme des objets dans la mesure où ils sont dénotables à l'aide des mêmes désignateurs linguistiques que les autres objets (SN définis, démonstratifs, pronoms anaphoriques...), mais appartiennent au domaine intensionnel dans la mesure où ils servent d'instruments de catégorisation par rapport aux realia, tout comme les fonctions.

\subsection{Le formatage ontologique de $<N>$ dans les anti ${ }_{1} N$}

Les formations en anti ${ }_{1} \mathrm{~N}$ mettent en évidence certaines difficultés d'interprétation que l'on trouve déjà au niveau de l'analyse des substantifs simples. Si la valeur sémantique adversative, attribuée à anti $i_{1}$, est relativement transparente, l'interprétation de l'opérande de $O P_{-} A N T I_{1}$, réalisé au niveau segmental par N, l'est bien moins : à un opérateur préfixal ayant une valeur sémantique relativement claire répond un $\mathrm{N}$ dont le "statut " est plutôt opaque. La question qui se pose à présent est de savoir à quel type d'entités on s'oppose, en se positionnant comme un anti ${ }_{1} \mathrm{~N}$. 


\subsubsection{Sous-spécification du formatage de $\langle\mathrm{N}\rangle$}

Certaines difficultés interprétatives des antiN relèvent du statut ambigu de $<\mathrm{N}>$ quant à sa catégorie ontologique: est-ce que le formatage de $<\mathrm{N}>$ est le même dans anti-Bush que dans anti-ours ? Et comment trancher? Dans la combinatoire syntaxique, les déterminants servent d'indicateurs pour spécifier le format de $<\mathrm{N}>$ (realia, type, etc.). Dans le cas des anti ${ }_{1} \mathrm{~N}$, le formatage de $<\mathrm{N}>$ est souvent sous-spécifié, certaines marques linguistiques de formatage ontologique, tels les déterminants par exemple, étant en principe absentes dans la combinatoire morphologique ${ }^{9}$ - ceci étant d'ailleurs vrai des mots construits en général (Temple, 1996). De ce fait, les emplois de anti ${ }_{1} \mathrm{~N}$ comportent donc souvent une part de flottement dû à la sous-spécification ontologique de $<\mathrm{N}>$. Dans les exemples suivants, le format de $<\mathrm{N}>$ dans anti-Ours n'est pas clair : s'agit-il d'un individu discret, d'un $\mathrm{N}_{\text {Type }}$ ou d'un $\mathrm{N}_{\text {classe }}$ ?

[14] Si vous êtes favorable à l'Ours, passez votre chemin, Jean-Pierre Pommièz est de toutes les manifestations anti-ours [...]. [w]

[15] Les anti-Ours manifestent dans les Pyrénées

[...] Quelque 5000 personnes venues des Pyrénées françaises mais également espagnoles ont manifesté à Bagnères-de-Bigorre, au sud de la France, contre l'introduction d'ours slovènes dans les Pyrénées. [w]

En [14], le SN défini singulier «l'Ours» ne renvoie pas à un ours particulier, mais à l'espèce; c'est ce que nous appelons à la suite de Berrendonner (2002) un type $\left(\mathrm{N}_{\text {Type }}\right)$. Dans l'expression anti ${ }_{1} \mathrm{~N}$, en revanche, le formatage de $\mathrm{N}$ est sous-spécifié : étant donné l'identité formelle entre le singulier et le pluriel, <ours> dans anti-ours peut à la fois renvoyer à un $\mathrm{N}_{\text {Type }}$ (glosé par "être opposé à l'ours») ou à un $\mathrm{N}_{\text {classe }}$ (glosé par «être opposé aux ours»), voire ni à l'un, ni à l'autre (voir infra, § 2.2). Le fait qu'en [14], on ait l'expression d'un $\mathrm{N}_{\text {Type }}$ (l'Ours) dans le contexte gauche de anti-ours et qu'en [15], il y a celle d'un $\mathrm{N}_{\text {classe }}$ (ours slovènes) dans le contexte droit de anti-ours ne peut servir d'indice pour trancher entre les deux lectures et il faut conclure à une sous-spécification du formatage de l'objet-de-discours <ours> dans anti-ours.

\subsubsection{Phénomènes de non-concordance en nombre entre substantif et anti ${ }_{1} \mathrm{~N}$ : interprétation $<$ Classe $>$ vs interprétation $<$ Type $>$}

Certains phénomènes de non-concordance en nombre entre le substantif régissant et l'épithète, comme en [16] et [17], sont des indices significatifs en ce qui concerne le formatage de $<\mathrm{N}>$ :

[16] Aux propriétés anti-ride et raffermissantes elle est idéale pour les peaux manquant de fermeté. [w]

[17] Bien choisir sa crème antirides

Qu'elles soient ridules, profondes, d'expression ou en " pattes d'oie ", les rides nous agacent $![\mathrm{w}]$

En [16], l'objet-de-discours <ride> est catégorisé comme un $\mathrm{N}_{\text {Type }}$, ce qui donne une glose en « qui agit contre la ride ». En [17] en revanche, on a affaire à un objet-de-discours qui est formaté comme un $\mathrm{N}_{\text {classe }}$ (i.e. " qui agit contre les rides ») ${ }^{10}$. Cette classe comporte différents éléments, comme en témoigne l'énumération à droite d'antirides, et peut être figurée comme suit : 
$[18]<$ rides $>=\{$ ridules, rides, pattes d'oie, ...\}

La même analyse vaut pour les exemples [19] et [20], <N> étant également formaté comme un $\mathrm{N}_{\text {classe }}$ :

[19] Elle a allumé une flamme anti-insectes dans un pot de grès,... [f, sOLLERS]

[20] Un collier anti-aboiements, baptisé sobrement «Aboistop», vient d'être mis au point à l'école nationale vétérinaire de Nantes [...]. [b2, Le Nouvel Observateur, 2 septembre 1988]

Comme le montrent les exemples [17], [19] et [20], le morphème -s dans les anti ${ }_{1} \mathrm{~N}$ n'est bien souvent pas tant une marque d'accord - ce qui éclairerait d'une lumière différente une affirmation de Rey (1968: 39), selon laquelle les anti ${ }_{1} \mathrm{~N}$ adjectivés n'auraient généralement pas la marque du genre et seraient par ailleurs assez souvent invariables qu'un indice servant à spécifier le format ontologique de $<\mathrm{N}>$.

\subsubsection{Interprétation extensionnelle de $<\mathrm{N}>$ dans les anti ${ }_{1} \mathrm{~N}$}

En dehors des anti ${ }_{1} \mathrm{~N}$ formés sur un nom propre (anti-Chirac, anti-Sarkozy) pour lequel le statut extensionnel de <Npropre> est plausible, les exemples où anti- opère sur un argument N', conçu comme une entité extensionnelle, sont relativement rares dans notre corpus. Soit l'exemple suivant, dans lequel le nom propre, en tant que désignateur direct, dénote l'objet-de-discours extensionnel <Nicolas Sarkozy> :

[21] Un journal « contre » Sarkozy mais « pour » quoi ? Est-ce suffisant de se positionner anti Nico le pas beau ? [w]

Dans cet exemple, la troncation familière du prénom et l'apposition «le pas beau » forcent une interprétation concrète. Voir également l'exemple suivant :

[22] Moi, je suis anti mère mais pas anti ma belle mère !! [...] Ma belle mère est très bien ma mère beurk. [w]

Dans anti ma belle mère, le possessif formate l'objet-de-discours <ma belle mère> clairement comme une entité extensionnelle, alors que le statut de <mère> dans anti mère reste potentiellement ambigu ( $\mathrm{N}_{\text {Type }}$ ou individu discret?), bien que le contexte favorise plutôt l'interprétation concrète (ma mère beurk). De nombreuses occurrences attestées, comme celles citées en [21] et [22] sont jugées agrammaticales par certains ; à notre avis, elles relèvent d'un phénomène de variation entre deux grammaires concurrentes emplois préfixal vs prépositionnel - du morphème anti ${ }_{1}^{-}$(Heyna, à paraître).

\subsection{Nbase dénote un objet-de-discours de type " attracteur »}

Bien souvent, on observe pourtant que les anti ${ }_{1} \mathrm{~N}$ construits sur un nom propre ne prennent pas forcément comme argument un objet existant in re, mais plutôt la politique du référent dénoté par $\mathrm{N}$, ses décisions, ses convictions, voire même ses partisans. Les anti-Sarkozy ne sont pas nécessairement opposés à Sarkozy en tant qu'« être en chair et en os ", mais ils sont opposés à sa façon de gouverner, à ses choix politiques, à l'UMP, etc. Idem pour les personnes qualifiées d'anti-Bush : 
[23] Tim Sebastian, qui a du mal à cacher ses sympathies pro-guerre, a mis sur la sellette le très anti-Bush-Blair, Georges Michael. [w]

En [23], <Bush> et <Blair> sont amalgamés en une seule entité, objet-de-discours auquel s'oppose Georges Michael : être anti-Bush-Blair, c'est être opposé à tout ce qui relève, d'une façon ou d'une autre, de $<$ Bush $>$ ou de $<$ Blair $>$. Ces emplois du nom propre constituent en quelque sorte un degré supplémentaire de la fonction référentielle étendue, réalisée par les métonymies intégrées qui rattachent un auteur à son œuvre, une personne au dossier la concernant, etc. (Kleiber 1990b, 1992) ${ }^{11}$.

On observe un phénomène similaire dans les occurrences d'anti-ours, citées ci-dessus en [14] et [15]. Les anti-ours ne détestent pas forcément les ours, mais sont opposés à l'introduction de l'ours dans une région donnée pour des raisons multiples (crainte des dégâts causés, brebis blessées, moutons égorgés, etc.) :

[24] Il est antiours lui aussi. Il explique qu'il est opposé au plan de réintroduction de la bête, mais qu'il n'en veut pas à la bête elle-même : « La Politique agricole commune, la PAC, fait plus de ravages que l'ours chez les éleveurs. Il n'y a plus assez de monde dans la montagne pour garder les troupeaux », rumine-t-il. [w]

Le fait de se positionner comme anti-ours permet d'activer toute une constellation de phénomènes qui entretiennent un rapport de contiguïté référentielle avec <ours> (Goodman 1985 ; Bonhomme 1987 ; Berrendonner 1990). Dans les formations de type antiours, $\mathrm{N}$ désigne donc un objet de connaissance fortement "implanté », au sens de Goodman (1985). Berrendonner (1990) utilise le terme d'attracteur pour désigner ce type d'objet-de-discours; les objets, événements et "épisodes locutoires» (ibid., 155), constitutifs d'un intertexte facilement accessible, fonctionnent comme des satellites, que l'attracteur regroupe autour de lui en raison d'un rapport référentiel étroit. Dans certains emplois de SN définis (singulier ou pluriel), l'objet-de-discours dénoté par le SN a le statut d'attracteur, comme l'illustrent les exemples ci-après :

[25] Slovène ou pas, peu importe... l'Ours divise la FRANCE ! Réintroduire ou ne pas réintroduire, that is the question. [w]

[26] L'ours, parfois, bouleverse les positions attendues. "Moi, je ne tirerai jamais sur lui ", annonce Arsène, l'antiours, dans un grand moment de paix. [w]

[27] <à propos d'associations de défense de l'environnement > les loups sont dans la rue. $[\mathrm{w}]$

Les emplois de SN définis singuliers commutent d'ailleurs généralement avec des paraphrases en l'affaire de $N$ ou la question de $N$ :

[28] Par le passé, la question de l'ours a été une affaire de spécialistes qui gardaient toutes les informations pour eux. [w]

Pour qu'un objet-de-discours puisse fonctionner comme attracteur, plusieurs conditions sont nécessaires (Berrendonner 1990). Nous retiendrons les suivantes : dans le cas de $<\mathrm{N}$, il doit non seulement s'agir d'un objet de connaissance fortement implanté, mais celui-ci doit également faire l'objet d'un débat et donc d'un ensemble de connaissances facilement accessibles ou présentes dans un contexte d'énonciation plus large. La réalisation de ces deux conditions explique la productivité de dérivés formés sur le nom 
propre d'un politicien (les anti-Chirac, les anti-Jospin, les anti-Sarkozy, etc.) ou sur des $\mathrm{N}$ qui désignent un référent faisant l'objet d'un débat dans l'actualité (cf. les débats autour du CPE, de l'introduction des ours ou des loups, etc.).

Citons un dernier exemple pour illustrer ce type de construction particulière :

[29] L'attitude des anti-arbres sur la place des Vosges est typique. Ce qu'ils aiment, c'est pas les endroits, c'est les décors. Tuer la vie pour restituer le décor. Ensuite, faire venir des figurants pour animer ce qu'on a tué. [b1, Le Nouvel Observateur, 9 février 1976]

Pour cet exemple, on peut également supposer que la plantation ou non d'arbres sur la place des Vosges ait fait l'objet d'un débat animé, d'où la possibilité de forger une expression anti $\mathrm{N}_{1} \mathrm{~N}$, dans laquelle $<\mathrm{N}>$ a le statut d'attracteur ${ }^{12}$.

L'observation qu'un objet-de-discours $<\mathrm{N}>$, réalisé sur le plan segmental par le substantif base, puisse faire office d'attracteur est plutôt surprenante, étant donné qu'il est généralement admis que les opérations dérivationnelles opèrent sur des lexèmes, i.e. des unités hors emploi (Fradin 2003).

Le fait que l'opérateur $O P_{-} A N T{ }_{1}{ }_{1}$ puisse prendre comme arguments des opérandes qui dénotent des objets-de-discours catégorisés tantôt comme $\mathrm{N}_{\text {Type }}$, tantôt comme attracteur crée des situations d'ambiguïté parfois volontaires et bienvenues. Ne pas devoir préciser à quoi on s'oppose concrètement ou pouvoir se rétracter, en refusant d'assumer certaines inférences, sont des facteurs qui permettent d'expliquer la productivité et la fréquence des dérivés anti ${ }_{1} \mathrm{~N}$.

\subsection{Le potentiel argumentatif des anti $\mathrm{N}_{1} \mathrm{~N}$}

Une première série de conclusions porte sur le pouvoir argumentatif des anti ${ }_{1} \mathrm{~N}$. Un atout non négligeable des dérivés de type anti ${ }_{1} \mathrm{~N}$ réside sans doute dans la concision de la forme, permettant l'association de formules prégnantes et brèves, illustrées par l'exemple et l'exemple ci-après :

[30] Qui est anti-marseillais ??

[...] tout le monde est anti marseillais-parisiens-lyonnais; du moment [où]

il supporte un autre club. [w]

A un niveau beaucoup plus fondamental, le pouvoir argumentatif des formations de type anti ${ }_{1} \mathrm{~N}$ relève de trois ordres de phénomènes pragmatico-discursifs :

(i) De façon générale, le format des objets-de-discours sur lesquels porte $O P_{-} A N T I_{1}$ n'est pas immédiatement accessible et nécessite le recours à des raisonnements inférentiels :

- Dans les anti ${ }_{1} \mathrm{~N}$, il peut y avoir hésitation sur le statut ontologique de l'objet-de-discours $<\mathrm{N}>: \mathrm{N}_{\text {Type }}$ ou attracteur, ce dernier résorbant tout ce qui va généralement avec le référent de $\mathrm{N}$ (i.e. un assemblage de choses).

- Lorsque le anti ${ }_{1} \mathrm{~N}$ est construit sur un nom propre, on retrouve ces mêmes ambiguïtés et il y a hésitation quant à savoir $\mathrm{si}<\mathrm{N}>$ est un objet réel, un type ${ }^{13}$, ou s'il fonctionne comme attracteur et dénote tout ce qui entretient un lien métonymique ou référentiel avec une personne donnée.

L'emploi de formations en anti ${ }_{1} \mathrm{~N}$ constitue donc une sorte de paradoxe dans la mesure où, par le biais de l'opérateur $O P_{-} A N I_{1}$, on affirme un positionnement idéologique ou 
axiologique clair - « être opposé à ", et en même temps, dans la même expression, l'objetde-discours dénoté par $\mathrm{N}$, en tant qu'opérande de $O P_{-} A N T I_{1}$, est extrêmement flou, voire indéterminé. Le pouvoir argumentatif des anti ${ }_{1} \mathrm{~N}$ réside à notre avis précisément dans cette contradiction.

(ii) Dans ce sens, on peut affirmer que le recours à un dérivé de type anti ${ }_{1} \mathrm{~N}$ constitue en quelque sorte une violation du principe de coopération, puisque la nature de $<\mathrm{N}>$ est sousspécifiée et que le décodeur ne dispose que de très peu de moyens pour savoir sur quoi précisément porte $O P_{-} A N T I_{1}$. Utiliser une expression en anti ${ }_{1} \mathrm{~N}$, c'est donc composer avec la maxime de nonchalance, que l'on glosera à la suite de Berrendonner (1990:150) par « Je me comprends, c'est l'essentiel ».

(iii) Si l'objet-de-discours $<\mathrm{N}>$ a bel et bien le statut d'attracteur au sens de Berrendonner (1990), le recours à un dérivé anti ${ }_{1} N$ permet d'évoquer confusément l'un ou l'autre satellite associé à $<\mathrm{N}>$ et donc de référer de façon nébuleuse. Il s'agit là d'une stratégie argumentative extrêmement puissante, qui - en omettant de spécifier à quoi précisément on s'oppose, et donc par cette indistinction même - laisse moins de prise à une contreargumentation.

\section{Les $\operatorname{anti}_{2} \mathbf{N}$ : un cas particulier de relation antonymique}

Les anti ${ }_{2} \mathrm{~N}$, dont antihéros ou antiroman ne sont que les exemplaires les plus immédiats, dénotent des objets-de-discours qui n'ont pas de référent existant in re, mais qui sont des entités idéelles ou des fictions mentales; de ce fait, les anti ${ }_{2} \mathrm{~N}$ appartiennent à la catégorie des types. Les anti ${ }_{2} \mathrm{~N}$ constituent en outre ce que G. Gross (1996:33) appelle une «nonprédication » dans la mesure où ils servent à désigner ou à nommer un référent et font donc partie du stock lexical au même titre que les mots simples ou composés.

\subsection{Les anti ${ }_{2} \mathrm{~N}$ - antonymes de quoi ?}

Dans les anti ${ }_{2} \mathrm{~N}$, anti $i_{2}^{-}$exprime une valeur antonymique (Rey 1968), également appelée descriptive (Corbin 1980) ou antipodale (Fradin 1997a). Que signifie pourtant «être à l'opposé de $\mathrm{N}$ » dans le cas des anti ${ }_{2} \mathrm{~N}$ ? Et quel est le rapport entre $\mathrm{N}$ et anti $\mathrm{N}$ (complémentarité, antonymie ou inclusion) ? La valeur antonymique de anti ${ }_{2}$ - n'est de loin pas une description sémantique précise... Dans certains cas, les anti ${ }_{2} \mathrm{~N}$ entrent en effet dans une relation d'ensemble complémentaire par rapport à $\mathrm{N}$ :

[31] Je crois que je suis un guerrier, et toi, que tu le veuilles ou non, tu es un anti-guerrier. [f, ABELLIO]

Dans d'autres cas de figure, assez fréquents, $\mathrm{N}$ et anti ${ }_{2} \mathrm{~N}$ sont prédiqués ensemble pour un même objet-de-discours :

[32] Notre hérö̈ne (ou plutôt anti-hérö̈ne) a 25 ans. Elle consacre son salaire à faire du shopping et à sortir avec sa meilleure amie. [p, Le Matin bleu , 30 mars 2007]

[33] Son aveu si caractéristique à Lady Melbourne, qu'il n'avance jamais que lorsque la femme a fait spontanément plus de la moitié du chemin, pourrait 
être la devise de cet anti-séducteur qui ne fait qu'un avec le séducteur malgré lui. [f, Du Bos]

Ces exemples semblent invalider une glose en «être l'opposé de $\mathrm{N}$ », puisqu'en principe anti ${ }_{2} \mathrm{~N}$ ne peut être valide en même temps que $\mathrm{N}$. La rectification montre que les anti ${ }_{2}$ $\mathrm{N}$ forment une sous-classe des $\mathrm{N}$, d'où un rapport d'inclusion logique, tout en entretenant un rapport d'antonymie avec la classe des $\mathrm{N}$.

Dans les anti ${ }_{2} \mathrm{~N}$, on retrouve également certaines difficultés interprétatives liées au formatage de $<\mathrm{N}>$, en particulier lorsque $\mathrm{N}$ est un nom propre (Jonasson 1994, Gary-Prieur 1994) : a-t-on affaire à un individu discret ou à un $\mathrm{N}_{\text {Type }}$ (i.e. $<\mathrm{N}>$ comme représentant typique d'une certaine vision du monde ou de la politique) ? L'exemple est parfaitement ambigu de ce point de vue :

[34] <à propos de Ségolène Royal> C'est à la fois l'anti-Sarkozy, l'anti-Chirac et l'anti-de Villepin. [w]

Dans cet exemple, il convient d'abord de clarifier à quel type de dérivés antiN on a affaire : la position "tête de $\mathrm{SN}$ » des dérivés antiN semble forcer une interprétation antonymique; en raison de l'article défini, l'hypothèse d'une substantivation d'un anti ${ }_{1} \mathrm{~N}$ paraît peu convaincante (cf. C'est une anti-Sarkozy, une anti-Chirac et une anti-de Villepin). Considérons le prédicat catégorisateur anti-Sarkozy, pour lequel plusieurs interprétations sont de prime abord en concurrence :

- <l'anti-Sarkozy> 䜿 « le contraire de Sarkozy » (<N> comme individu, existant in re)

- <l'anti-Sarkozy> 屁 « le contraire du type d'hommes politiques incarné par Sarkozy» $(<\mathrm{N}>$ comme type, existant in intellectu)?

Dans cette dernière interprétation, on aurait affaire à un cas particulier de métonymie, le nom propre servirait à dénoter un certain type de convictions politiques. Cette interprétation semble se rapprocher de celle d'anti-sarkozysme, dont le sens est moins opaque.

Autre exemple :

[35] Il est naturel qu'un anti-Barrès ait succédé ici à Barrès, comme il est souhaitable qu'un anti-Gide succède à Gide. [f, Collectif, Arts et littérature dans la société contemporaine]

Le nom propre Barrès remplit sa fonction référentielle et désigne un individu concret. Dans anti-Barrès en revanche le nom propre fait office de catégorisateur dans la mesure où il représente un certain nombre de valeurs ${ }^{14}$; le Npr fonctionne dès lors comme un type. Une des valeurs sémantiques de $a n t i_{2}$ - en tant qu'opérateur semble donc consister à formater l'objet-de-discours dénoté par le nom propre comme un $\mathrm{N}_{\text {Type }}$, et non plus comme un individu discret.

La présence du morphème -s force une interprétation $\mathrm{N}_{\text {classe }}$, pluralisation d'un $\mathrm{N}_{\text {Type }}$ :

[36] Il y aura un Maeterlinck xxii et il y a des Verlaines et des anti-Verlaines comme les papes et les antipapes. [f, GIDE, VALÉRY]

Là encore, les dérivés anti ${ }_{2} \mathrm{~N}$ dénotent des objets-de-discours qui appartiennent au domaine intensionnel et non pas au domaine des réalités concrètes. 


\subsection{Anti $_{2}$ - comme opérateur de sous-catégorisation}

Corbin (1980: 196) se contente d'observer au sujet des anti ${ }_{2} \mathrm{~N}$ que « le sens du dérivé est l'opposé de celui du radical ; dérivé et radical forment un couple antithétique, où anti- a une valeur d'opposition "descriptive" $»^{15}$. La valeur d' " antonymie » se manifeste à la fois dans les dérivés de type antiroman que dans les dérivés comme antimoral, les deux types de formation étant caractérisés par un «non-changement catégoriel du radical » (ibid.). En guise d'illustration, elle glose deux exemples : « un antiroman est un roman dont les caractéristiques sont opposées à celles du roman traditionnel, un comportement antimoral est un comportement dont les manifestations sont opposées à celles d'un comportement moral » (ibid.). Corbin décrit implicitement le sens d'antiroman et d'antimoral comme découlant d'un calcul à partir d'une référence, qui serait « le roman traditionnel », ou « un comportement moral », sans donner plus de précisions.

Fradin (1997a: 90) est à ce sujet plus explicite: un lexème anti ${ }_{2} \mathrm{~N}$ dénote «un objet caractérisé par le fait qu'il se définit par la négation des propriétés stéréotypiquement attachées à N ", d'où le recours à l'étiquette d'antipodaux qu'il emprunte à Lyons (1977 : 207-335). Dans (1997b : 340), Fradin observe à juste titre que «anti- n'opère pas sur la représentation sémantique du $\mathrm{N}$ prise en bloc mais au contraire sur chaque propriété qu'encode cette dernière ». Il en donne une illustration avec l'exemple d'anti-Madonna, que nous allons commenter infra (\$3.3). Les faits empiriques confirment que $\mathrm{OP}_{-} A N T I_{2}$ opère par conséquent sur les propriétés intensionnelles de N' et annule certaines de ses qualités prototypiques.

Amiot (2004: 70) observe au sujet des noms dérivés d'un préfixe de type 2 (anté-, anti-, co-, extra-, etc.) que

[...] le nom dérivé (désormais $\mathrm{Nd}$ ) désigne toujours une entité de même nature que ce qui est dénoté par le nom base (désormais $\mathrm{Nb}$ ), celle-ci se différenciant de l'entité à laquelle réfère le $\mathrm{Nb}$ par un certain nombre de propriétés, plus ou moins élevé : la relation entre le $\mathrm{Nd}$ et le $\mathrm{Nb}$ peut être une relation d'altérité totale (comme dans la préfixation par anti-) [...].

Il n'est pas très facile de savoir ce qu'il faut entendre par " entité de même nature », si ce n'est que $\mathrm{Nd}$ et $\mathrm{Nb}$ désignent tous deux un référent qu'on peut approximativement qualifier de $\mathrm{N}$ (ex. un antihéros est une sorte de héros); on ne peut en revanche être d'accord avec l'idée que le préfixe anti $i^{-}$exprime une "altérité totale». La particularité sémantique de $\mathrm{OP}_{-} A N \mathrm{AT}_{2}$ consiste précisément dans le fait d'exprimer à la fois une appartenance à la classe des N' et un écart par rapport au prototype exprimé par N’.

Plus généralement, on peut supposer que les anti ${ }_{2} \mathrm{~N}$ entretiennent une relation de type hyponyme/hyperonyme avec $\mathrm{N}$ : les antihéros forment une sous-catégorie de la classe des héros, les antiromans appartiennent - malgré l'écart souvent volontaire d'avec le prototype - au genre romanesque. Dans ces dérivés, l'opérateur $O P_{-} A N T I_{2}$ a une fonction déterminative, voire «typologisante» (cf. G. Gross 1996: 51), étant donné qu'il apporte une spécification ou une sous-catégorisation dans le cadre d'une taxinomie. Cette valeur «typologisante » de $O P_{-} A N T I_{2}$ apparaît clairement dans le test d'effacement du préfixe : alors qu'un antihéros reste malgré tout une sorte de héros, les dérivés préfixés par antin'entretiennent pas cette relation avec le $\mathrm{N}$ dont ils sont dérivés, un antimite n'étant pas une sous-catégorie de mites. 
La relation particulière entre $\mathrm{N}$ et anti ${ }_{2} \mathrm{~N}$ explique également pourquoi il est possible de prédiquer ensemble $\mathrm{N}$ et anti ${ }_{2} \mathrm{~N}$ pour un même objet-de-discours :

[37] Notre héroïne (ou plutôt anti-héroïne) a 25 ans. Elle consacre son salaire à faire du shopping et à sortir avec sa meilleure amie. [p, Le Matin bleu, 30 mars 2007]

[38] Son aveu si caractéristique à Lady Melbourne, qu'il n'avance jamais que lorsque la femme a fait spontanément plus de la moitié du chemin, pourrait être la devise de cet anti-séducteur qui ne fait qu'un avec le séducteur malgré lui. [f, DU BOS]

La rectification en [37] et la double prédication anti-séducteur et séducteur malgré lui en [38] montrent bien que les anti ${ }_{2} \mathrm{~N}$ forment une sous-classe des $\mathrm{N}$; de ce fait, ils entrent dans rapport d'inclusion logique, tout en entretenant un rapport différentiel avec la classe des N.

\section{3. $A n t i_{2}$ - comme « modifieur d'intension »}

Il s'agit à présent de cerner plus précisément ce rapport différentiel induit par anti ${ }_{2}^{-}$. Sans vouloir chercher une description sémantique dans les paraphrases de dictionnaires qui se contentent de gloser les anti ${ }_{2} \mathrm{~N}$ par «être à l'opposé de $\mathrm{N}$ », il importe de se poser la question de ce que faut-il entendre par «être à l'opposé de $\mathrm{N}$ ». La nature du rapport entre $\mathrm{N}$ et anti ${ }_{2} \mathrm{~N}$ est plus complexe qu'un rapport de simple antonymie.

\subsubsection{L'opérateur anti ${ }_{2}$ - modifie l'intension de $\mathrm{N}^{\prime}$}

Les candidats au statut de "base dérivationnelle» des anti ${ }_{2} \mathrm{~N}$ sont des $\mathrm{N}$ qui appartiennent à des paradigmes taxinomiques très divers (littérature, philosophie, etc.). Plus généralement, les $\mathrm{N}$ dans les anti ${ }_{2} \mathrm{~N}$ appartiennent à la catégorie des noms de types et dénotent donc des objets-de-discours intensionnels. Quant aux dérivés anti ${ }_{2} \mathrm{~N}$, ils permettent de dénoter des objets-de-discours qui ne comportent pas tous les traits prototypiques ${ }^{16}$ inscrits dans le signifié du $\mathrm{N}_{\text {Type }}$. Le préfixe anti $i_{2}$ - peut par conséquent être décrit comme un opérateur qui agit sur l'intension de N', en annulant ou en renversant certains des traits sémantiques considérés comme prototypiques. Selon Rey (1968: 53), anti $i_{2}^{-}$opèrerait un renversement vers le domaine non-prestigieux : "Antiaffecte le contenu analysable (sèmes) de prestige d'un exposant qui les supprime (nonprestige) ou les renverse ». Les faits empiriques montrent qu'un renversement axiologique est souvent réalisé, mais n'est de loin pas systématique [cf. 45].

Dans sa fonction de "modifieur d'intension », OP_ANTI ${ }_{2}$ prend comme opérande N' dont il annule certains traits intensionnels. Pourtant, suffisamment de traits pertinents sont maintenus, pour que le référent appartienne malgré tout à la catégorie générale des $\mathrm{N}$. Soit schématiquement ${ }^{17}$ :

$$
\begin{aligned}
& \text { [39] } N^{\prime}=\{\mathrm{s} 1, \mathrm{~s} 2, \mathrm{~s} 3 \ldots \mathrm{sn}\} \quad \text { (traits pertinents « positifs ») } \\
& \operatorname{anti}_{2} \mathrm{~N}^{\prime}=\{-\mathrm{s} 1,-\mathrm{s} 2, \ldots \mathrm{sn}\} \quad \text { (mêmes traits « négativisés ») }
\end{aligned}
$$

La relation entre $\mathrm{N}$ et anti ${ }_{2} \mathrm{~N}$ est donc à la fois une relation d'antonymie (traits inversés) et une relation d'inclusion logique (structure sémique identique) : $\mathrm{N}$ et $\operatorname{anti}{ }_{2} \mathrm{~N}$ partagent un certain nombre de sèmes communs; par contre, les anti ${ }_{2} \mathrm{~N}$ comportent en outre des 
sèmes « négativisés » ou « renversés » de $\mathrm{N}$. Sur le plan extensionnel, l'extension du anti ${ }_{2}$ $\mathrm{N}$ (c'est-à-dire la classe des objets catégorisés comme des anti ${ }_{2} \mathrm{~N}$ ) est incluse dans l'extension de $\mathrm{N}$. De ce fait, il est à notre avis inapproprié de parler d'antonymie dans le cas des $a_{n} i_{2} \mathrm{~N}$ - ni l'antonymie complémentaire de type pair/impair ou fille/garçon, ni l'antonymie scalaire de type chaud/froid ne semblent adéquates ${ }^{18}$.

\subsubsection{L'opérateur anti ${ }_{2}$ - prend comme opérande certains traits prototypiques de $\mathrm{N}^{\prime}$}

L'opérateur $\mathrm{OP}_{-} \mathrm{ANTI}_{2}$ annule bien certains traits prototypiques, sans qu'il ne soit jamais précisé quelles sont les propriétés qui sont en quelques sortes annulées. Selon Fradin (1997b : 340), « la préfixation d'anti- affectera les propriétés stéréotypiques à l'exclusion des autres ", c'est-à-dire à l'exclusion des propriétés constitutives (P). Selon Fradin, la représentation sémantique d'anti-Madonna pourrait donc être schématisée comme suit :

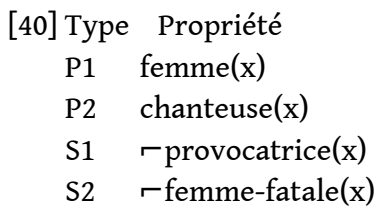

Tout d'abord, il convient de noter que les emplois d'anti-Madonna à valeur adversative sont majoritaires, si on se base sur les occurrences trouvées sur la toile. Dans les emplois d'anti-Madonna à valeur antonymique, on constate que le anti ${ }_{2} \mathrm{~N}$ est effectivement toujours utilisé pour qualifier des femmes chanteuses :

[41] <à propos de Björk> Star de la pop, star de cinéma, sacrée meilleure actrice à Cannes pour le rôle de Selma dans la comédie musicale « Dancers in the Dark » de Lars von Trier, dont elle a écrit la musique, l'Islandaise est une des grandes divas du XXIe siècle. Une sorte d'anti-Madonna. Alors que l'Américaine joue tout sur l'apparence, la récupération (intelligente) des modes et des sons "tendance ", l'Islandaise, elle, invente l'avant-garde, la diffuse et la popularise (elle a d'ailleurs écrit une chanson pour la Madone...). [w]

Comme dans l'exemple cité, il n'est en revanche souvent pas décidable quelles sont les propriétés stéréotypiques qui sont annulées.

Reprenons à présent un exemple cité en introduction :

[42] Scandaleux Mercutio, conteur de balivernes, antihéros que n'anime nul esprit de sacrifice non plus qu'aucune dévotion à quelque cause juste ou injuste, mais qui se risque par jeu! [f, LEIRIs].

Dans cet extrait, certains traits intensionnels prototypiques de ce que devrait être un héros sont explicités et explicitement niés, d'autres sont rajoutés (se risquer par jeu) pour justifier le prédicat catégorisateur d'antihéros. En se basant sur l'extrait de Leiris, les intensions de héros et d'antihéros peuvent être représentées comme suit :

[43] héros $=\{\mathrm{s} 1$ [+esprit de sacrifice], s2 [+dévotion à une cause $], \ldots\}$ antihéros $=\{-\mathrm{s} 1,-\mathrm{s} 2, \mathrm{~s} 3[+ \text { se risquer par jeu }], \ldots\}^{19}$

Prenons un exemple que nous avons déjà cité (supra, ) : 
[44] <à propos d'une BD manga> Voilà une série hors du commun, le héros Dark Schneider est totalement antihéros, cruel, sadique, pervers et sans aucune moralité. [w]

Dans cet emploi d'antihéros, ce ne sont pas les mêmes traits qui sont renversés, comme le montre la suite d'adjectifs qualificatifs (cruel, sadique, pervers et sans aucune moralité), qui sert de glose pour élucider le terme d'antihéros.

\subsection{La fréquence des gloses accompagnant les anti ${ }_{2} \mathrm{~N}$}

De façon quasi systématique, on trouve des indices dans l'entourage proche du dérivé qui corrobore l'hypothèse que $O P_{-} A N T{ }_{2}$ agit sur l'intension de N'. Le fait qu'on ne puisse pas déterminer sur quels traits porte précisément l'opérateur $O P_{-} A N T_{2}$ explique la forte présence de paraphrases descriptives qui servent justement à expliciter l'écart par rapport au prototype dénoté par N. C'est ce mécanisme qui a permis d'expliciter l'emploi d'antihéros en [2] et [44]. Voici un autre exemple :

[45]<à propos de Rosemonde, créatrice de colliers> Elle aime le jade, particulièrement le jade ancien de Chine [...]. Parfois, elle en fait le pendentif d'un de ces grands colliers agrémentés de grosses perles ou de lourdes pierres. Septante de ces créations récentes, qu'elle nomme aussi anticolliers parce qu'ils sont parfois difficiles à porter vu leur poids, sont exposés à Lausanne. [p, Fémina, 7 novembre 2004, p. 11]

Un collier vise à être porté, et c'est précisément ce trait qui est renversé ou négativisé par OP_ANTI ; la glose explicative en "parce que..." justifie le recours à un prédicat catégorisant de type anti ${ }_{2}$ N. Autre exemple :

[46] <à propos des Chroniques romanesques de Giono> Nous sommes donc face à une dérive du roman policier, et nous entrons dans le monde du roman d'un justicier. Pour autant, très vite les circonstances vont faire qu'il s'agit en fait d'un anti-roman policier. En effet, ce n'est pas Langlois qui retrouve le coupable, mais Frédéric II, par hasard, et non pas au terme de longues recherches, élaborées. [w]

Les Chroniques romanesques de Giono sont qualifiées d'anti-roman policier parce que le coupable est retrouvé par hasard, et même pas par celui qui mène l'enquête. Là encore, un connecteur (En effet) établit le lien entre l'emploi d'un anti ${ }_{2} \mathrm{~N}$ et les justifications de cet emploi. Voir également l'exemple suivant :

[47] Margaret Thatcher est l'anti-Robin des Bois, a remarqué Gordon Brown, député travailliste, elle prend aux pauvres pour donner aux riches. [b2, L'Evénement du Jeudi, 21 avril 1988]

En tant qu'opérateur, $O P_{-} A N T I_{2}$ instaure donc souvent une relation paradoxale d'appartenance à une catégorie et de différenciation ou de distanciation par rapport à cette même catégorie. Cette dualité sémantique explique la forte présence de gloses dans le contexte étroit des anti ${ }_{2} \mathrm{~N}$; il s'agit là d'une caractéristique récurrente des emplois de anti ${ }_{2} \mathrm{~N}$. 


\section{Conclusions}

\subsection{Les objets-de-discours sélectionnés par anti ${ }_{1}$ - et anti ${ }_{2}^{-}$}

Le préfixe anti $i_{1}$ - opère préférentiellement sur des objets-de-discours existants in intellectu : les $<\mathrm{N}>$ sélectionnés par le préfixe anti ${ }_{1}$ - sont catégorisés majoritairement comme des types ou des concepts (anti-cléricalisme). Dans notre corpus, nous avons relevé très peu d'exemples où $O P_{-} A N T I_{1}$ prend comme argument un objet existant in re. Même dans les cas de figure où le dérivé anti ${ }_{1} \mathrm{~N}$ est construit sur un nom propre, ce dernier ne dénote bien souvent pas l'objet existant in re, mais fonctionne comme attracteur (comme en [23]) pour signifier confusément une opposition face aux agissements de la personne dénotée par le nom propre, ou alors le nom propre sert d'instrument de catégorisation et dénote un type (ainsi anti-Barrès et anti-Gide en [35]), le dérivé est alors interprété comme un anti ${ }_{2} \mathrm{~N}$ à valeur antonymique.

Certains emplois atypiques, mais très répandus en français contemporain corroborent l'observation que anti $i_{1}$ - sélectionne préférentiellement des objets intensionnels : OP_ANTI, dans une certaine variété de français, opère sur des expressions catégorisantes, qui peuvent prendre diverses formes :

(i) des SN suivis d'une relative déterminative, qui a pour fonction de sous-catégoriser un groupe de personnes :

[48] La xénophobie est la peur des étrangers. Toi tu balances carrément des discours grotesques anti-les-gens-pas-de-la-même-couleur. [w]

(ii) des arguments complexes de la forme [N+épithète]:

[49] En marge, Serge avait écrit : "Arroum, arrêté le 28 mars ». La fiche de Redouane se terminait par cette conclusion : " Depuis son retour, n'a participé qu'à des actions anti-dealers d'héroïne. Pas encore assez fiable, semble-t-il. Mais à surveiller. N'a plus aucun repère. Très encadré par Nacer et Hamel. [f, Izzo]

Une exception à la sélection préférentielle d'objets intensionnels est pourtant également attestée : les anti-[proSN] appartiennent aux realia.

[50] Le pire c'est les anti-français, ils sont anti eux-mêmes ! [w]

[51] Boris et moi aurions voulu cracher sur la vermine anti-nous... [f,

SCHREIBER]

L'immense majorité des emplois confirme néanmoins que le préfixe anti- semble spécialisé dans l'expression de l'opposition face à un objet-de-discours formaté comme un $\mathrm{N}_{\text {Type }}$, et donc plus généralement comme un objet idéel.

Le préfixe anti $i_{2}$-rejoint sur ce point son homonyme, puisqu'il sélectionne également des $\mathrm{N}_{\text {Type }}$. Sa fonction sémantique est toutefois différente, puisqu'il fait office à la fois d' opérateur de sous-catégorisation au sein d'un type (d'où un rapport d'inclusion logique) et de modifieur d'intension (d'où un rapport d'antonymie avec $\mathrm{N}$ '). Les objets-de-discours dénotés par anti ${ }_{2} \mathrm{~N}$ entretiennent une relation originale avec l'objet dénoté par $\mathrm{N}$, puisqu'ils sont à la fois hyponyme et antonyme de N'. Il reste que la portée de l'opérateur $\mathrm{OP}_{-} A N T I_{2}$ sur le 
plan sémantique n'est pas clairement identifiable, étant donné qu'il négativise ou annule certains traits de N', considérés comme typiques, sans préciser lesquels.

\subsection{Les anti ${ }_{1} \mathrm{~N}$ et les anti ${ }_{2} \mathrm{~N}$ : deux types de structurations internes}

L'examen des mécanismes de formation n'ayant pas été notre objectif ici, nous n'aborderons que brièvement certains aspects en lien avec les phénomènes dérivationnels. Les préfixes anti $i^{-}$et anti $i_{2}^{-}$s'appliquent tous les deux à des bases substantivales (antirides vs antiroman) et adjectivales (antisceptique vs antijoli< TLFi). Le préfixe anti ${ }_{1}$ - construit des noms (adjectifs-substantifs) sur une base substantivale. Les anti ${ }_{1} \mathrm{~N}$ sont par conséquent des constructions exocentriques ${ }^{20}$, qui sont tantôt utilisés adjectivement (contextes $\alpha$ et $\gamma$ ), tantôt substantivement (contexte $\beta$ ). Lorsqu'ils sont utilisés substantivement, ils prennent le genre masculin par défaut. Le préfixe anti ${ }_{2}^{-}$en revanche crée des substantifs à partir d'une base substantivale; les anti ${ }_{2} \mathrm{~N}$ sont par conséquent endocentriques. En ce qui concerne le genre des $\operatorname{anti}_{2} \mathrm{~N}$, il est toujours identique à celui de leur base.

Pour expliquer la différence sémantique entre antirides et antiroman, nous avancerons l'hypothèse que le morphème anti- possède une seule valeur, mais entre en fait dans deux structurations internes différentes, que l'on peut décrire en termes de relation de dépendances à la suite de Hjelmslev (1968-1971). Dans les deux types de dérivés, on a affaire à un rapport de dépendance unilatérale, l'orientation du rapport de dépendance n'est par contre pas la même dans les deux cas de figure :

$$
\begin{aligned}
& \text { [52] } \operatorname{anti}_{1} \mathrm{~N}: \text { anti- } \leftarrow(\mathrm{N}) \text { ex. antirides } \\
& \operatorname{anti}_{2} \mathrm{~N}: \quad \text { (anti-) } \rightarrow \mathrm{N} \quad \text { ex. antihéros }
\end{aligned}
$$

Dans les dérivés anti ${ }_{1} \mathrm{~N}, \mathrm{~N}$ est régime de anti $i_{1^{-}}$, tandis que dans les anti ${ }_{2} \mathrm{~N}$, c'est l'inverse : anti $i_{2}$ - est régime de $\mathrm{N}$, puisque dans ce contexte, $\mathrm{N}$ et anti ${ }_{2} \mathrm{~N}$ peuvent commuter et que $\mathrm{N}$ peut par ailleurs figurer seul. Plusieurs arguments corroborent cette hypothèse :

- Du point de vue catégoriel, la base nominale et le dérivé anti ${ }_{2} \mathrm{~N}$ appartiennent tous les deux à la catégorie des substantifs, alors que ce n'est pas le cas pour les anti ${ }_{1} \mathrm{~N}$, qui sont catégorisés comme des noms (adjectifs-substantifs).

- Le test de la suppression confirme cette analyse à la fois sur le plan syntaxique et sur le plan du contenu : dans les anti ${ }_{2} \mathrm{~N}$, la suppression de anti- est possible, étant donné que les anti ${ }_{2} \mathrm{~N}$, tout comme les $\mathrm{N}$ dont ils sont dérivés, appartiennent au paradigme des substantifs catégorisateurs (les antihéros sont des héros et les antiromans sont des romans), d'où le statut de modifieur d'intension de $O P_{-} A N T I_{2}$. Cf. les manipulations suivantes :

[53] [...] très vite les circonstances vont faire qu'il s'agit en fait d'un antiroman policier. [exemple cité en ]

$v s[. .$.$] très vite les circonstances vont faire qu'il s'agit en fait d'un roman$ policier.

A l'inverse, la suppression de anti $i_{1}^{-}$'est pas possible dans les anti ${ }_{1} \mathrm{~N}$, sans changer complètement le statut catégoriel et fonctionnel de $\mathrm{N}$ :

[54]Elle a allumé une flamme anti-insectes dans un pot de grès [...]. [f, SOLLERS] vs 'Elle a allumé une flamme insectes dans un pot de grès [...]. 
[55] Les anti-ours des Pyrénées et les anti-loups des Alpes se sont rencontrés. [w]

vs Les ours des Pyrénées et les loups des Alpes se sont rencontrés

L'énoncé modifié en [54] pose problème quant à son interprétation (que peut être une flamme insectes?). L'exemple [55] illustre bien que $\mathrm{N}$ et anti ${ }_{1} \mathrm{~N}$ ne forment pas un paradigme : ours et loups sont toujours des substantifs catégorisateurs, alors que anti-ours et anti-loups peuvent être analysés ici comme des qualificatifs à droite d'un nom zéro ou comme des prédicats catégorisateurs.

Pour expliquer cette différence de comportement sémantique de anti-, deux approches celles de Amiot (2005) et de Van Goethem (2007) - nous paraissent pertinentes. Amiot (2005) utilise les termes d'endocentrique vs exocentrique pour décrire la différence structurelle entre certains dérivés et composés ${ }^{21}$. Dans un cas comme avant-scène, le sens est endocentrique, c'est-à-dire que le dérivé est un hyponyme de $\mathrm{N}$ (comme dans nos dérivés anti ${ }_{2} \mathrm{~N}$ ); il hérite par ailleurs le genre du substantif base. Dans d'autres cas, comme dans avant-guerre ${ }^{22}$, sans-abri ou après-midi, le sens est exocentrique ou « prédicatif » et le fonctionnement de l'élément formateur est tout à fait identique à celui de la préposition (ce qui correspondrait aux dérivés de type anti ${ }_{1} \mathrm{~N}$ ) ; pour ce second type de composés, le dérivé est généralement masculin pour les inanimés (genre par défaut), masculin ou féminin pour les animés.

Van Goethem (2007) distingue deux types de composés du point de vue sémantique :

- d'une part, les composés exocentriques ayant la structure sémantique «Tête-Modifieur " ${ }^{23}$ ( sur-mesure, faire du sur-place) ;

- d'autre part, les composés endocentriques à structure sémantique "Modifieur-Tête » ( surcapacité, surnom).

On pourrait rapprocher le fonctionnement des antiN de cette analyse. Dans les anti ${ }_{1} \mathrm{~N}$, anti- aurait le statut de "Tête » au même titre qu'une préposition; dans les anti ${ }_{2} \mathrm{~N}$, antiferait office de «Modifieur» et apporterait une modification intensionnelle au sens du radical.

\section{BIBLIOGRAPHIE}

AMIOT, D. 2004. Préfixes ou prépositions ? Le cas de sur(-), sans(-), contre(-) et les autres. Lexique 16 : 67-83.

AмIOT, D. 2005. Between compounding and derivation: Elements of word formation corresponding to prepositions. Morphology and its Demarcations. Selected Papers from the 11th Morphology Meeting, Vienna, February 2004, W. U. DRESSLER et al. (éds.). Amsterdam/Philadelphia: John Benjamins. 183-195.

BERRENDONNER, A. 1990. Attracteurs. Cahiers de linguistique française $11: 149-158$.

BERRENDONNER, A. 1995. Quelques notions utiles à la sémantique des descripteurs nominaux. Tranel $23: 9-39$. 
BERRENDONNER, A. 2002. Types. Les facettes du dire : hommage à Oswald Ducrot, M. CAREL (éd.). Paris :

Kimé. 39-53.

BERRENDONNER, A. 2004. Intensions et extensions. Structures et discours : mélanges offerts à Eddy

Roulet, A. Auchlin et al. (éds.). Québec : Nota bene. 151-165.

BonHomme, M. 1987. Linguistique de la métonymie, Berne : Peter Lang.

CORBIN, D. 1980. Contradictions et inadéquations de l'analyse parasynthétique en morphologie

dérivationnelle. Théories linguistiques et traditions grammaticales, A.-M. DESSAUX-BERTHONNEAU (éd.).

Lille : Presse universitaire de Lille. 181-224.

DUCROT, O. 1973. La preuve et le dire : langage et logique, Paris : Mame.

FRADIN, B. 1997a. Esquisse d'une sémantique de la préfixation en anti-. Recherches linguistiques de Vincennes $26: 87-112$.

FRADIN, B. 1997b. Une préfixation complexe : le cas de anti-. Neuphilologische Mitteilungen XCVIII/4 : 333-349.

FRADIN, B. 2003. Nouvelles approches en morphologie, Paris : Presses universitaires de France.

GALLIOT, M. 1955. Essai sur la langue de la réclame contemporaine, Paris : E. Privat.

GARY-PRIEUR, M.-N. 1994. Grammaire du nom propre, Paris : Presses universitaires de France.

GOODMAN, N. 1984. Faits, fictions et prédictions, Paris : Minuit.

GROSS, G. 1996. Les expressions figées en français : noms composés et autres locutions, Gap : Ophrys

Collection L'Essentiel du français.

HEYNA, F. à paraître. The use of anti- in contemporary French: a case of degrammaticalization?

HEYNA, F. en cours. Analyse morphosyntaxique de phénomènes dérivationnels 'atypiques' : les formations parasynthétiques revisitées. Thèse de Doctorat. Fribourg $(\mathrm{CH})$ : Université de Fribourg.

HJELMSLEV, L. 1968-1971. Prolégomènes à une théorie du langage, Paris : Minuit.

JONASSON, K. 1994. Le nom propre : constructions et interprétations, Louvain-la-Neuve : Duculot.

KLEIBER, G. 1981. Problèmes de référence : descriptions définies et noms propres, Paris : Klincksieck.

KLEIBER, G. 1990a. La sémantique du prototype : catégories et sens lexical, Paris : Presses universitaires de France.

KLEIBER, G. 1990b. Paul est bronzé vs La peau de Paul est bronzée. Contre une approche référentielle analytique. Analyse et synthèse dans les langues slaves et romanes. $V^{e}$ Colloque International de linguistique slavo-romane : 109-134. Tübingen : G. Narr.

KLEIBER, G. 1992. Qui est sur l'étagère de gauche? ou faut-il multiplier les référents ? Travaux de linguistique et de philologie 30 : 107-124.

LYONS, J. 1970. Linguistique générale. Introduction à la linguistique théorique. Paris : Larousse.

LYONS, J. 1977. Semantics, Cambridge: Cambridge University Press.

LYONS, J. 1978. Eléments de sémantique, Paris : Larousse.

NOAILlY, M. 1990. Le substantif épithète, Paris : Presses universitaires de France.

NOAILLY, M. 1999. L'adjectif en français, Gap : Ophrys Collection L'Essentiel du français.

REY, A. 1968. Un champ préfixal : les mots français en anti-. Cahiers de lexicologie XII-1 : 37-57. 
TEMPLE, M. 1996. Pour une sémantique des mots construits, Villeneuve d'Ascq : Presses universitaires du Septentrion.

VAN GOETHEM, K. 2007. L'interaction entre la grammaticalisation et la structure du mot : l'emploi lié de la préposition sur, communication lors du colloque Autour de la préposition, 20-22 septembre 2007. Caen : Université de Caen.

\section{NOTES}

1. Nous n'avons pas pu consulter l'article de J. Durand (1982), «A propos du préfixe anti- et de la parasynthèse en français ", Occasional Papers from the Language Centre 25, University of Essex, 175-208.

2. Les exemples sont essentiellement issus de la presse écrite [p] (en partie grâce au moteur de recherche GlossaNet) et d'Internet [w] ; pour ces derniers, l'orthographe a été normalisée. Ces données sont complétées par quelques exemples oraux [o] et des occurrences tirées des bases textuelles Frantext [f] et Bornéo 1 et 2 [b].

3. Dans une perspective diachronique relativement étroite, il intéressant de constater une évolution importante quant à la fonction attributive des dérivés que nous avons appelés anti ${ }_{1} \mathrm{~N}$. En 1968, Alain Rey observe (1968: 41) que les anti ${ }_{1} \mathrm{~N}$ peuvent figurer en position attribut et présente ceci comme une nouveauté par rapport à l'analyse de Galliot en 1955 ; en revanche, il note que les dérivés en anti- ne prennent pas - «(ou pas encore)» comme il le précise - les degrés de comparaison. Une recherche sur corpus montre clairement que la position d'attribut est tout à fait répandue pour les anti ${ }_{1} \mathrm{~N}$, ainsi que la présence de modifieurs (très, assez, adverbes en -ment, etc.).

4. Cf. l'usage traditionnel des étiquettes latines : nomen substantivum, nomen adjectivum (Noailly, 1999 : 13). Noailly observe à ce propos : «Plutôt que d'adjectif et de substantif, il serait sage de parler de comportement adjectival ou de comportement substantival, sachant que les unités nominales du lexique français sont souvent aptes (et volontiers disposées) à passer d'un rôle à l'autre. » (ibid.).

5. Corbin emprunte à Ducrot (1973) les qualificatifs « descriptifs » vs " polémiques », appliqués initialement à la négation.

6. Fradin renvoie à Lyons (1977) : les antipodaux se caractérisent par la négation des propriétés stéréotypiques attachées à $\mathrm{N}$.

7. Jonasson (1994: 64-72) distingue trois emplois prototypiques du nom propre: 1) la fonction référentielle (J'ai écrit à Paul.), 2) la fonction vocative (Pierre! Ecoute-moi.), et 3) la fonction de nomination qui regroupe la nomination didactique (Mon frère s'appelle Paul.) et la nomination performative (Je te baptise Thérèse.).

8. Pour Berrendonner (2002: 50 ), l'existence des types démontre qu'il y a non-coïncidence entre l'opposition objet/fonction et entre l'opposition extension/intension.

9. En français contemporain, les $\mathrm{SN}$ définis à droite de anti $i_{1}^{-}$sont tout à fait courants, non seulement sur le web, mais également dans la littérature contemporaine et dans la presse écrite (« un discours anti-les méchants libéraux»; "le patch anti rondeurs disgracieuses » $[w]$ ) (Heyna, à paraître).

10. Selon Berrendonner (2002 : 51), les classes appartiennent à l'univers extensionnel : «L'idée que tout prédicat aurait une "référence virtuelle » me semble ainsi, pour tout dire, une pseudosystématisation scolastique suspecte, qui fait peu de cas des conditions réelles d'emploi du langage. »

11. Cf. le célèbre exemple : George Sand est sur l'étagère de gauche. 
12. Dans cet exemple, la portée de $O P_{-} A N T I_{1}$ n'est pas tout à fait claire : il n'est pas exclu que l'opérateur adversatif porte sur l'expression «arbres sur la place des Vosges». Certains faits authentiques laissent penser que $O P_{-} A N T I_{1}$ peut prendre comme opérande une énonciation (Heyna en cours).

13. Cf. l'exemple [35].

14. Cf. en particulier les emplois attributifs des noms propres (Jonasson, 1994 : 85ssq.).

15. Corbin renvoie à Ducrot (1973: 123-131) pour les qualificatifs « descriptif » vs " polémique », appliqués initialement à la négation. Dans l'annexe 8 (1987:652), elle réunit les deux effets de sens dans une seule règle sémantique, étant donné que tous les noms construits sur une base nominale peuvent théoriquement réaliser et un sens oppositif et un sens descriptif.

16. Nous référons à la notion de prototype telle que la définit Kleiber (1990a: 49) : «Le prototype est ainsi conçu comme étant le meilleur exemplaire communément associé à une catégorie. ».

17. Les notations sont inspirées de Rey (1968: 53).

18. Cf. les sous-catégories introduites par Lyons (1978: 218ssq.) : les antonymes au sens strict, les contraires, les converses et les oppositions directionnelles.

19. Nous nous contentons ici de citer les traits caractéristiques tels qu'ils figurent dans l'exemple, sans les transposer dans le cadre de l'analyse sémique traditionnelle.

20. Les termes d'endocentrique et d'exocentrique sont issus de la terminologie de Bloomfield, définis par Lyons (1970 : 179) comme suit : «On dira qu'une construction est endocentrique quand sa distribution est identique à celle d'un ou plusieurs de ses constituants; toute construction qui n'est pas endocentrique est exocentrique. (Autrement dit, l'exocentricité est définie négativement par rapport à l'endocentricité et toutes les constructions appartiennent nécessairement à l'une ou à l'autre de ces catégories) ».

21. Selon Amiot (2005: 185), dérivés et composés sont traditionnellement considérés comme endocentriques (tête droite), les lexèmes exocentriques et/ou à tête gauche étant formés en syntaxe.

22. Amiot (2005: 189) introduit la notion de "weak endocentricity " pour les composés à valeur temporelle, formés sur avant- et après- : ces termes ne sont pas prédicatifs au même titre que les sans $N$, étant donnés qu'ils réfèrent à une période, alors que $\mathrm{N}$ désigne un événement (avant-mai 68 ) ou un actant lié à une période (avant-Ceaucescu/après-Ceaucescu). La notion d'attracteur, exposée ci-dessus (\$2.2.2), permettrait à notre avis d'intégrer ces formations au sein des composés exocentriques.

23. Précisons que Van Goethem s'écarte du classement des constructions proposé par Lyons $(1970: 180)$ et Bloomfield. Lyons utilise les termes "tête-modificateur " pour décrire une souscatégorie des endocentriques, à savoir les endocentriques par subordination de types $(A+N)$ ou $(\mathrm{Adv}+\mathrm{A})$, dont la distribution est identique à celle de leur constituant principal.

\section{RÉSUMÉS}

Cette étude examine les dérivés dénominaux en anti- du point de vue sémantique et argumentatif. Deux types de dérivés sont discriminés en fonction de la valeur sémantique que prend anti-: valeur adversative dans les anti ${ }_{1} \mathrm{~N}$ (ex. anti-rides, antigrippe), valeur antonymique dans les anti ${ }_{2} \mathrm{~N}$ (ex. antihéros, antiroman), l'apparition de l'une ou l'autre valeur étant étroitement liée aux contextes syntaxiques dans lesquels figurent les dérivés antiN. Dans les anti ${ }_{1} \mathrm{~N}$, on 
s'intéresse dans un premier temps au format ontologique de l'objet-de-discours $<\mathrm{N}>$ : celui-ci est tantôt catégorisé comme un individu discret, comme un type (i.e. un objet intensionnel) ou comme une classe, tantôt il a le statut d'un « attracteur » (Berrendonner 1990), tantôt encore, le format ontologique de $<\mathrm{N}>$ est sous-spécifié. A partir de faits authentiques, on montre dans un second temps que le potentiel argumentatif des anti ${ }_{1} \mathrm{~N}$ réside précisément dans ces diverses possibilités de formatage, y compris dans la sous-spécification de $<\mathrm{N}>$. Pour le deuxième type de dérivés, les anti ${ }_{2} \mathrm{~N}$, la notion d'antonymie est approfondie. A l'issue de cet article, une hypothèse est avancée sur le type de structure interne des deux types de dérivés.

The aim of this study is to examine antiN derivatives from a semantic and a pragmatic point of view, especially as far as argumentative possibilities are concerned. Two types of derivatives can be distinguished depending on the semantic content of the prefix: anti海 takes an adversative meaning in anti1N derivatives (eg. anti-rides, antigrippe) and an antonymous meaning in anti2N derivatives (eg. antihéros, antiroman). It will be shown that the interpretation of the antiN derivative is closely related to the syntactic contexts in which it figures. For the anti1N derivatives, the ontological category of $<\mathrm{N}>$ will be examined (intensional or intensional object, "attracteur" (Berrendonner 1990), etc.). Within a corpus-based approach, it will be argued that the under-specification of $<\mathrm{N}>$ might account for the argumentative potential of the anti1N derivatives. For the second type of derivatives, i.e. anti2N derivatives, the antonymic relation will be more clearly defined. Finally, a hypothesis about the internal structure of the two types will be made.

\section{INDEX}

Keywords : antonymy, argument types, argumentation, dependences, endocentric construction, exocentric construction, extension, intension, lexical negation, prefix anti-, prototype semantics, type

Mots-clés : antonymie, argumentation, attracteur, classe, endocentricité, exocentricité, extension, formatage ontologique, intension, négation lexicale, préfixe anti-, relation de dépendances, sémantique du prototype, type

\section{AUTEUR}

\section{FRANZISKA HEYNA}

Université de Friburg \& ATILF (Nancy université - CNRS)

Séminaire de linguistique française, Av. de Beauregard 13, CH-1700 Fribourg

franziska.heyna@unifr.ch 